\title{
INVERSION OF ELECTRICAL RESISTIVITY TOMOGRAPHY DATA DERIVING FROM 3D STRUCTURES
}

Tsourlos P.

Department of Geophysics, School of Geology, Aristotle University of Thessaloniki, 54124, Thessaloniki, Greece tsourlos@lemnos.geo.auth.gr

\section{ABSTRACT}

In this work the effectiveness of $2 \mathrm{D}$ and $3 \mathrm{D}$ algorithms for inverting Electrical Resistivity Tomography (ERT) data deriving from 3D structures is studied. Further, an analysis of data-collection strategies in the case of 3D structures is being carried out. Dense 2D measurements are considered a practical tool for mapping 3D structures given the current limitations in ERT hardware.

To perform the tests $2 \mathrm{D}$ and a $3 \mathrm{D}$ inversion programs are used. Both schemes use a forward model based on a 2.5D and 3D finite element scheme respectively. For both the $2 \mathrm{D}$ and $3 \mathrm{D}$ cases a fully non-linear inversion scheme based on a smoothness constrained algorithm is used. The Jacobian matrix is calculated using the adjoined equation technique.

Comparisons are being carried out by means of synthetic examples for $3 D$ models and dense 2D measurements with their axis parallel to the $X$ ( $X$-lines) and/or $Y(Y$-lines) directions. For the case of $3 D$ structures and $2 D$ inversion tests results illustrate that both $X$-line, $Y$-line measurements are required to delineate the modeling body. However, when 3D inversion is considered either $X$ line or $Y$-line measurements are adequate to produce good quality reconstructions of the subsurface.

Overall, results clearly illustrate the superiority of 3D over 2D inversion schemes in the case of $3 \mathrm{D}$ structures both in view of quality and logistics. Despite the increased computational time required by $3 \mathrm{D}$ inversion schemes, good quality results can be produced. Further, 2D inversion techniques require effectively a double amount of measurements to produce acceptable results. The ongoing advancement of fast computers renders the described approach of combining dense 2-D measurement with $3 \mathrm{D}$ inversion practical for routine data treatment.

\section{INTRODUCTION}

The Electrical resistivity tomography (ERT) technique is considered as one of the most significant new geophysical methodologies to emerge in the last decade. ERT is now widely used for environmental, engineering and groundwater resource mapping.

The development of the technology associated with automatically multiplexed electrode arrangements and automatic measuring systems facilitate the acquisition of a large number of measurements in a limited time. Further, the advent of fast computers allowed the development of the automated resistivity inversion schemes which aim to construct an estimate of a subsurface resistivity distribution which is consistent with the experimental data. Among others, the smoothness constraint inversion (Constable, 1987) has become the most popular for interpreting ERT data since it produces a simplified subsurface resistivity model which is a reasonable representation of the subsurface and at the same time guarantees inversion stability.

Several 2D smoothness constrained inversion algorithms for ERT data have been presented in literature (Sasaki 1989, Xu 1993, Elis \& Oldenburg 1994, Tsourlos 1995, Loke \& Barker 1996a).

Further, since many of the problems associated with geophysical exploration are of a threedimensional nature several algorithms for treating the ERT problem in three-dimensions have been presented (among others Park \& Van 1991, Loke \& Barker 1996b, Tsourlos \& Ogilvy 1999). 
Despite the development of advanced instrumentation and 3D interpretation techniques still common geophysical practice relies mainly on 2D approaches both in view of measurements and interpretations (Chambers, 2001). Even in the case of fully 3D resistivity variations 2D measurements are still quite popular among geophysicists. In this framework the effectiveness of 2D and 3D inversion strategies for the case of 3D structures is studied in the present work on the basis of synthetic model tests in combination to data-collection strategies. To perform the tests $2 \mathrm{D}$ and $3 \mathrm{D}$ inversion programs are used. Both schemes use a forward model based on the finite element method, whereas inversion is based on the same, fully non-linear, iterative scheme.

(a)

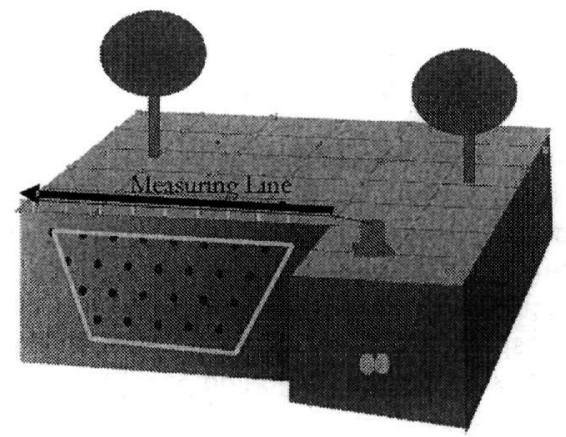

(b)

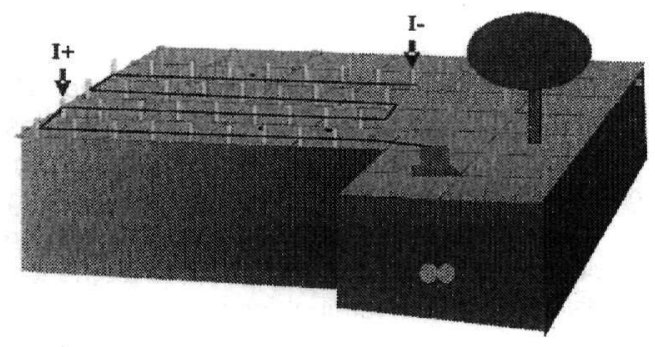

Figure 1: a) 2D measuring mode, b) 3D measuring mode

\section{MEASURING-INTERPRETATION STRATEGY}

There are two main measuring modes in ERT: a) 2D measuring mode involves laying a number of equally spaced electrodes over a line and obtain measurement combinations for varying spacing in order to obtain sectional images of the subsurface (Fig 1a) and b) 3D measuring mode which involves laying out a number of electrodes on a 3D grid and measure a large number 4-electrode combinations in order to obtain information about the 3D variation of the subsurface resistivity (Fig 1b). 2D measuring mode is fast, easy and low-cost to implement, and results can be interpreted fast using $2 \mathrm{D}$ inversion programs but is problematic when $3 \mathrm{D}$ structures are to be detected. Conversely $3 D$ measuring mode treats successfully $3 D$ structures however it is expensive since it involves increased instrumentation (cable) and computational cost if data are to be treated with 3D inversion programs. The later is not an important problem considering the high increase in computer power but hardware logistics is most of the times prohibitive in measuring with the 3D mode particularly with large electrode spacing.

So in practice instead of using the 3D measurement mode 3D resistivity variations are recorded by recording a dense 2D measurement grid which is considered to be a more practical and economical approach for field-data. Dense $2 \mathrm{D}$ sets involve measuring parallel $2 \mathrm{D}$ lines with inter-line spacing equal to the inter-electrode spacing. Measurements can take place along the $X$-axis $(X$ lines) (Fig 2a), or along the $Y$ axis ( $Y$-lines) (Fig 2b), or along both axes ( $X Y$-lines) as depicted in figure $2 \mathrm{c}$.

These dense $2 \mathrm{D}$ measurements are routinely being interpreted with $2 \mathrm{D}$ algorithms and the results are combined a-posteriori to generate pseudo-3D $(x, y, z)$ images. This type of results often suffer from artifacts either due to the fact that $3 \mathrm{D}$ responses are attributed to $2 \mathrm{D}$ structures and/or due to the varying level of misfit that individual $2 \mathrm{D}$ inversions may reach to. It is therefore reasonable to assume that the quality of the pseudo-3D images is dropping the noisier the data and the more complicated (3D) the subsurface structure is. Techniques such as smoothing can certainly improve pseudo 3D results yet this approach is not implied by the data or the model but is simply a mathematical operation lacking physical (geological) intuition. 
(a)

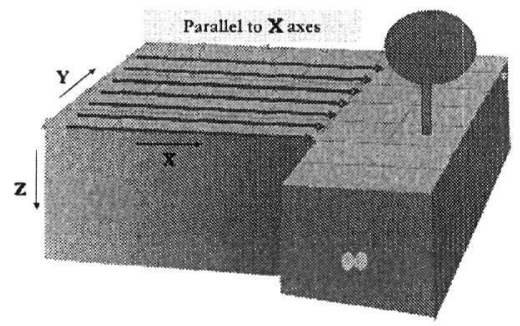

(b)

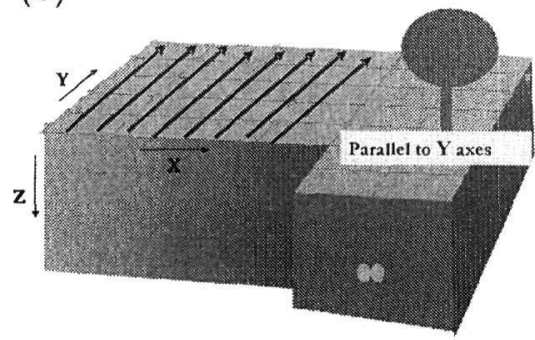

(c)

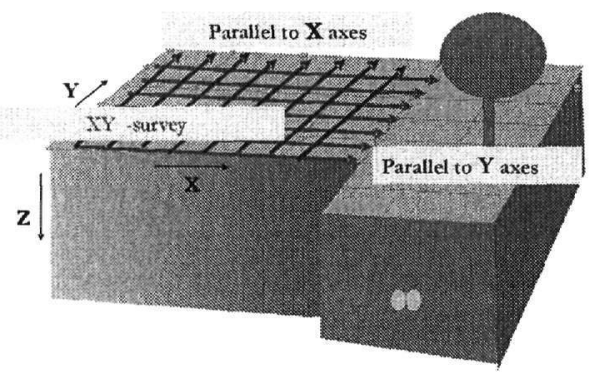

Figure 2: Dense 2D measurements a) parallel to the $X$-axis ( $X$-lines), b) parallel to the $Y$-axis $(Y$-lines), c) combined $X Y$-lines.

\section{MODELLING AND INVERSION}

The models presented in this work have been calculated using the finite element method (FEM). The technique has been extensively described in many works so only a brief description of the method is presented here.

\subsection{5-D Forward Modelling}

In $2.5 \mathrm{D}$ modelling the change in resistivity is considered to be two dimensional but the current flow pattern is a three dimensional one. In other words, the measured values correspond to a three dimensional subsurface where the resistivity is allowed to vary in only two dimensions and remains constant in the strike direction (Tsourlos et al., 1999). To include the potential variability in the strike (y) direction a cosine Fourier transformation is applied.

The FEM treats the problem by discretizing the earth into homogeneous triangular (in this case) regions called elements (Fig 3 a). The potential within each element is approximated by a simple interpolation function (basis function). In order to minimize the error between the approximated and real potential, the Galerkin minimization criterion is applied. After applying the Galerkin minimization scheme to every element, the individual element equations can be assembled in to one global system which has the following form:

$$
K A=F \text {, }
$$

where $A$ is the unknown transformed nodal potential vector, $F$ is the vector describing the sources and $\mathrm{K}$ is a matrix which is related to the nodal coordinates. After applying the homogeneous Dirichlet and Newman boundary conditions the system of equation (1) is being solved and the transformed nodal potential is obtained. After solving equation (1) for several wavenumbers the total potential is recovered by applying the inverse Fourier transform. Since the nodal potential is known, point to point potential differences and apparent resistivities are easily obtained. 


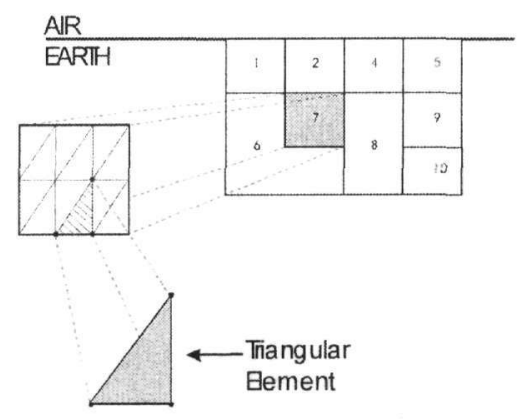

hexahedral element

Figure 3: (a) triangular element - 2.5D FEM, (b) hexahedral element -3D FEM.

\subsection{D Forward Modeling}

The approach is very similar to the $2.5 \mathrm{D}$ case however here the problem is treated in $3 \mathrm{D}$ so there is no need to use a fourier transformation. The solution of the differential equation that governs the flow of the electrical current in the ground (Poisson equation) is sought by subdividing the area into hexahedral elements (Fig $3 b$ ). After applying the Galerkin minimization scheme to every element, the individual element equations are assembled to form the global system which has the form of equation 1.

The final step is to solve the system of equations: for the 3D case which in general involves large systems of equations an iterative technique is preferable. In this work the conjugate gradient method for solving large sparse linear systems is used.

\subsection{Inversion}

The inversion core algorithm is the same for both the 2D and $3 \mathrm{D}$ case. A non-linear smoothness constrained inversion algorithm was used (Sasaki, 1992). The inversion is iterative and the resistivity $x k+1$ at the $k+1$ th iteration is given by:

$$
\mathbf{x}_{k+1}=\mathbf{x}_{k}+d \mathbf{x}_{k}=\mathbf{x}_{k}+\left[\left(\mathbf{W}_{\mathrm{d}} \mathbf{J}_{k}\right)^{\top}\left(\mathbf{W}_{\mathrm{d}} \mathbf{J}_{\mathrm{k}}\right)+\mu_{\mathrm{k}}\left(\mathbf{C}_{\mathrm{x}}^{\top} \mathbf{C}_{\mathrm{x}}+\mathbf{C}_{\mathrm{z}}^{\top} \mathbf{C}_{\mathrm{z}}\right)\right]^{-1}\left(\mathbf{W}_{\mathrm{d}} \mathbf{J}_{\mathrm{k}}\right)^{\top} \mathbf{W}_{\mathrm{d}} \mathrm{d} \mathbf{y}_{\mathrm{k}}
$$

where $C_{x}, C_{z}$ are matrices which describe the smoothness pattern of the model in the $x$ and $z$ axes respectively (de Groot-Hedlin and Constable, 1990), dy $y_{k}$ is the vector of differences between the observed data $\mathbf{d}^{\text {obs }}$ and the modeled data $\mathbf{d}_{k}{ }^{\text {calc }}$ (calculated using the forward modeling technique $2.5 \mathrm{D}$ or $3 \mathrm{D}$ ), $\mathrm{J}_{\mathrm{k}}$ and $\mu_{\mathrm{K}}$ is the Jacobian matrix estimate and the Lagrangian multiplier respectively for the $k^{\text {th }}$ iteration, $\mathbf{W}_{d}$ is the diagonal matrix of the data variances, and $T$ denotes the transpose. The adjoint equation approach (McGillivray \& Oldenburg, 1990) was incorporated into the FEM scheme in order to calculate the Jacobian matrix J (Tsourlos, 1995). Depending on the dimensions of our problem the Jacobian matrix is calculated either by the $2.5 \mathrm{D}$ or the 3D forward solver.

\section{SYNTHETIC EXAMPLES}

Synthetic data were created assuming a $15 \times 15 \times 8$ 3D grid. Synthetic data involved measuring $152 \mathrm{D}$ lines parallel to the $X$-axis and $152 \mathrm{D}$ lines parallel to the $Y$ axis. Inter-line and inter-electrode spacing was set to be $a=5 \mathrm{~m}$. Synthetic data were obtained using the pole-dipole array with max_n separation equal to $7 a$. 


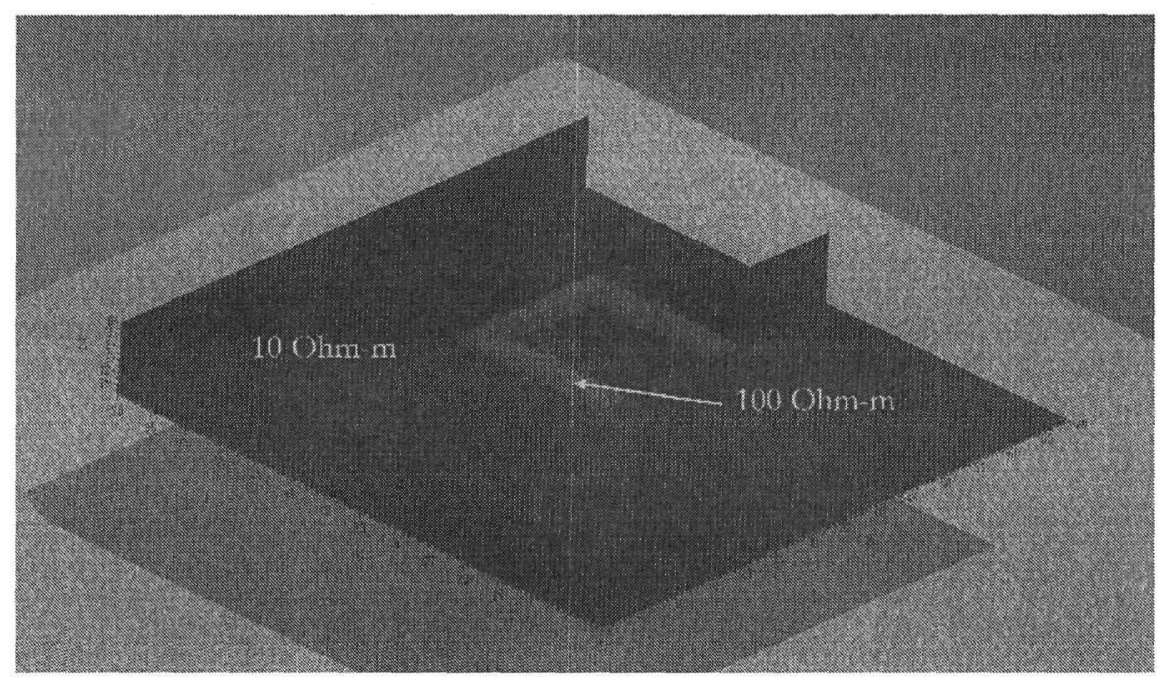

Figure 4: Model 1

\section{MODEL 1}

The modeling body is a typical 3D structure and is depicted in figure 4 . The produced synthetic data were inverted using 2D inversion and the interpreted 2D sections were combined to produce pseudo-3D depth slices for the $X$-lines, $Y$-lines and $X Y$-lines and are depicted in figure 5 . It is clear that in the $X$-lines inversion the features parallel to the $X$ axis cannot be reconstructed. $A$ similar result is obtained for the $Y$-lines and the features parallel to the $Y$ axis. Only when both $X, Y$-lines are inverted the modeled body is reconstructed successfully. This signifies that in dense $2 D$ surveys if $2 D$ interpretation is to be used both $X, Y$-lines need to be measured and combined in order to be safe that no feature is "missed".

However this is not the case when data are inverted using the $3 D$ inversion scheme. In figure 6 it is clearly shown that the $3 D$ inversion results plotted as depth slices are equally satisfactory for the $X$-lines and $X Y$-lines and superior to the $2 D$ inversion results for the $X Y$-lines. This signifies that if 3D inversion is used measuring just the $X$ or the $Y$ lines is enough to produce good quality results.

\section{MODEL 2}

The synthetic data (X,Y,XY-lines) produced for the modeling body of figure 7 were inverted using $2 D$ inversion. Results are depicted in Figure 8 . In this case, although the $2 D$ inversion of $X Y$ lines is superior to the $X$ and $Y$ lines inversions, the latter managed to delineate the modeling body far more successfully than the previous case. This is due to the fact that the modeling body has a dip in relation to the measuring axes. This signifies that in areas where the main strikes of the targets are known (i.e faulting systems) and the survey orientation dips the main strikes, 2D inversion of just the $\mathrm{X}$ or $\mathrm{Y}$ lines is enough to obtain an acceptable subsurface image.

\section{MODEL 3}

The synthetic data ( $X, Y, X Y$-lines) produced for the modeling body of figure 9 were inverted using both $2 \mathrm{D}$ and $3 \mathrm{D}$ inversion. Results are depicted as depth slices in figure 10 . All inverted images $(2 D, 3 D)$ are in general satisfactory however again $3 D$ inversion results are by far more accurate Also note that the $3 D$ inversion results of the $X$ and $X Y$-lines are effectively identical. In figure 11 a $3 \mathrm{D}$ view of $\mathrm{X}$-lines $3 \mathrm{D}$ inversion results is depicted 


\section{CONCLUSIONS}

The results illustrate the superiority of 3D over the pseudo-3D inversion schemes. Dense $2 D$ measurements across either $X$ or $Y$ axis combined with 3D inversion schemes are adequate to delineate even complicated 3D structures. The 2D (Pseudo-3D) schemes requires measurements across both $X-Y$ axes in order to produce safe information.

This approach of combining dense 2-D measurement with 3D inversion is considered practical for routine data treatment since the extra computational time/power required by 3D inversion schemes is compensated by the reduced amount ( $50 \%$ less) of field data required when compared with the 2D approach.

MODEL $Z=1.25 \mathrm{~m}$

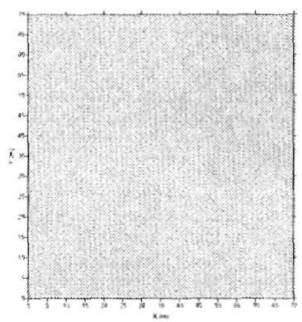

MODEL $\quad Z=3.75 m$

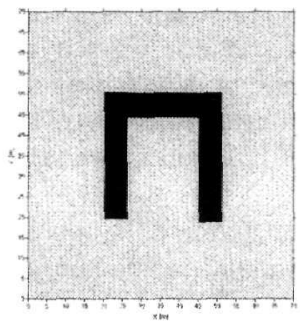

2D XY INVERSION
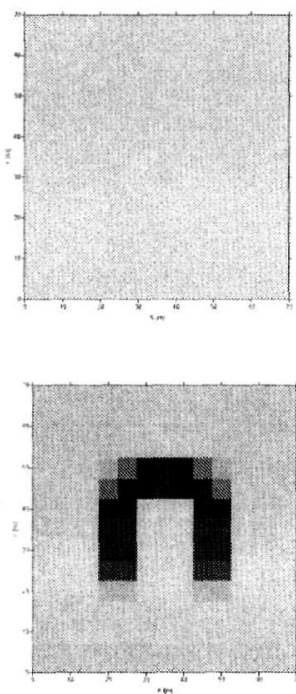

2D X INVERSION
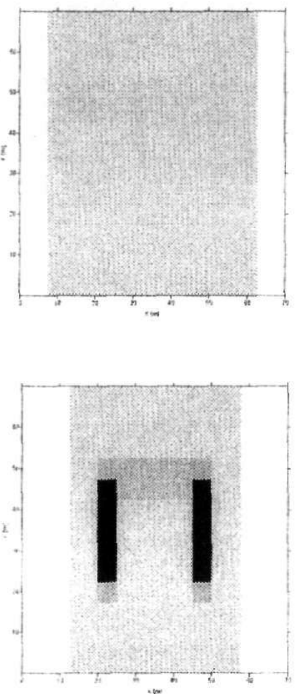

2D Y INVERSION
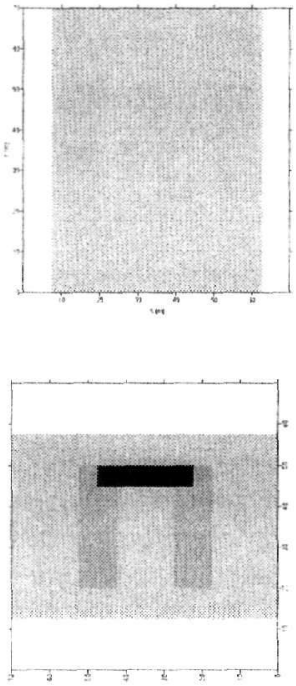

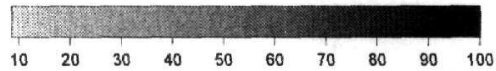

Figure 5: Pseudo-3D depth slices of the 2D inversions for the $X$-lines, $Y$-lines and $X Y$-lines of Model 1

MODEL $Z=3.75 m$

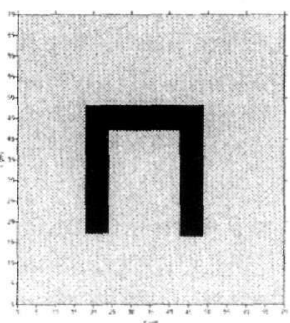

2D XY INVERSION

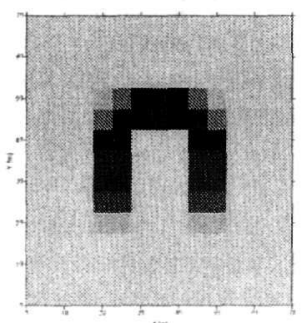

3D XY INVERSION

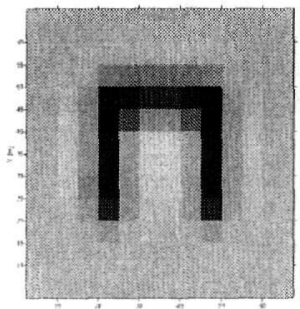

3D X INVERSION

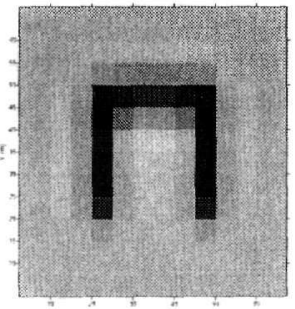

Figure 6: $2 D$ and 3D inversion results for the $X$-lines and $X Y$-lines of Model 1 presented as depth slices 


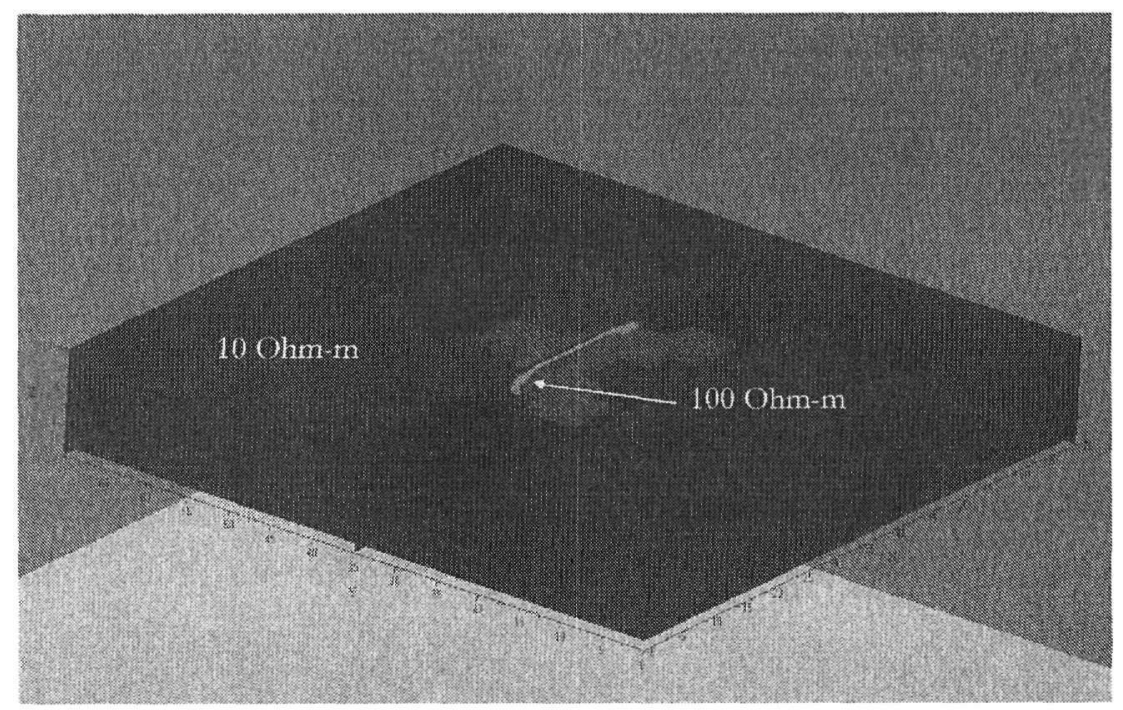

Figure 7: Model 2

MODEL $Z=3.75 \mathrm{~m}$

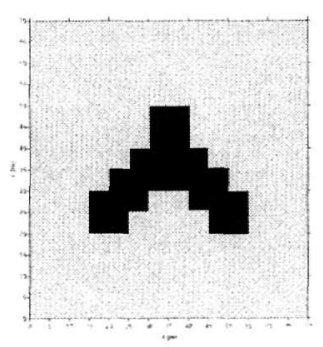

MODEL $Z=6.75 \mathrm{~m}$

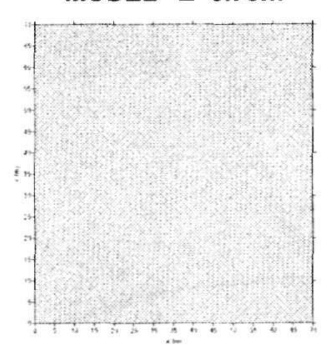

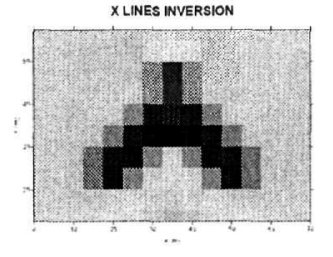
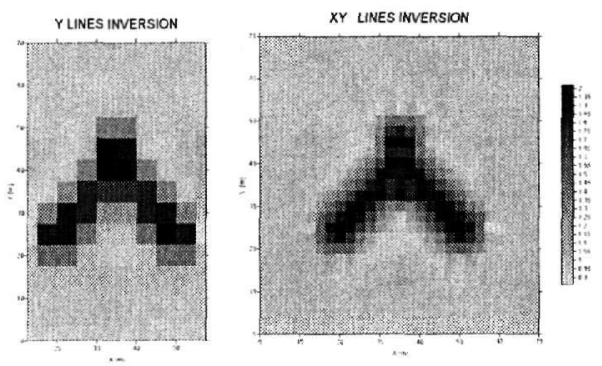

XY LINES INVERSION
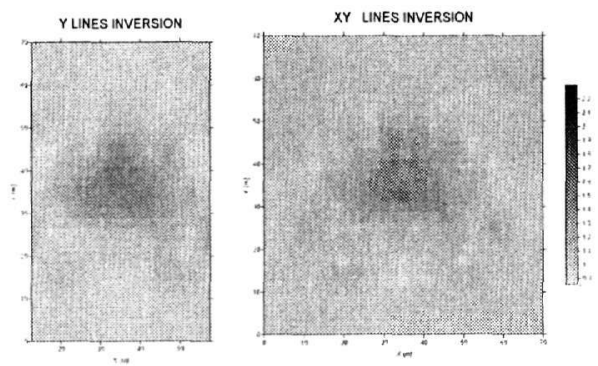

Figure 8: Pseudo-3D depth slices of the $2 D$ inversions for the $X$-lines, $Y$-lines and $X Y$-lines of Model 2 


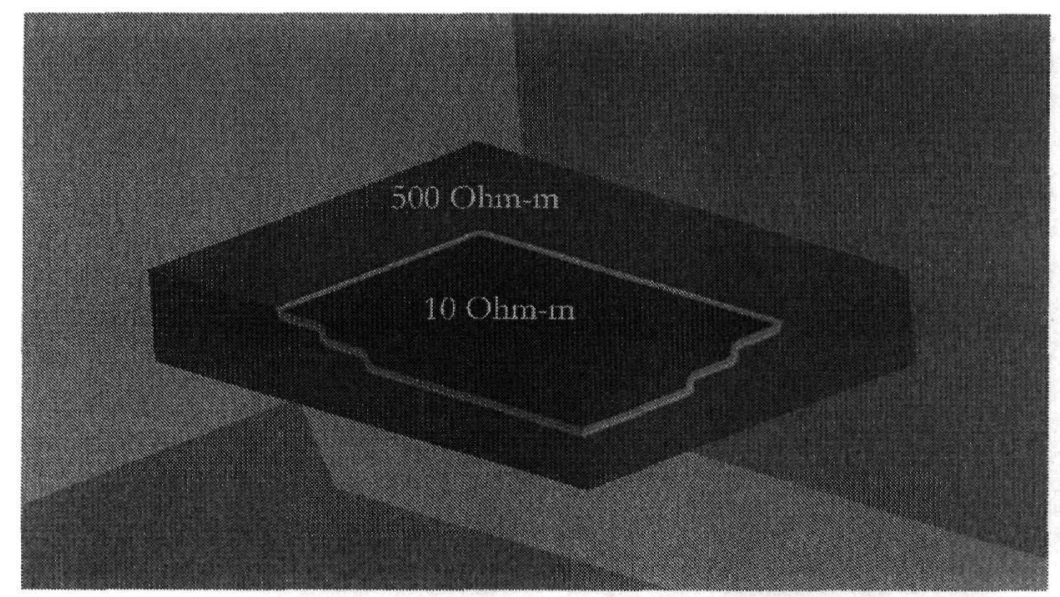

Figure 9: Model 3
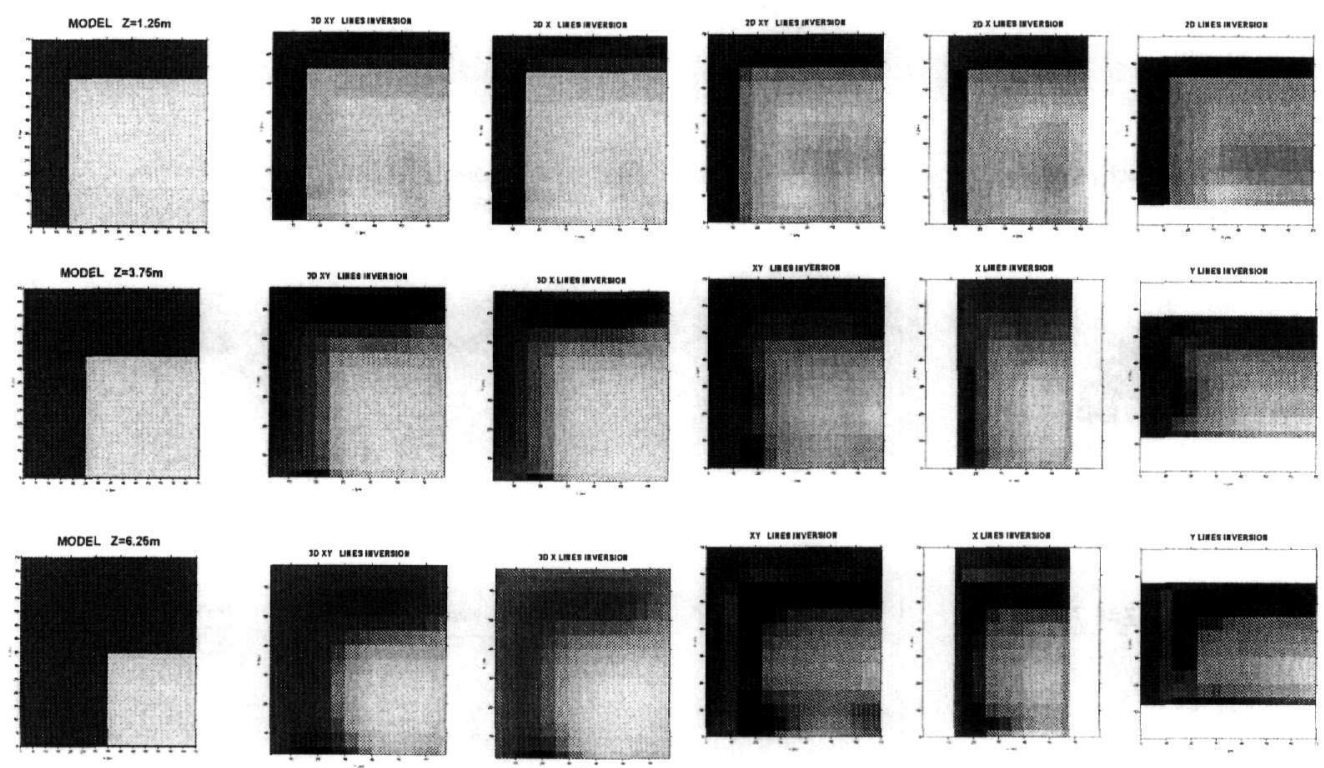

MODEL $z=8.75 \mathrm{~m}$
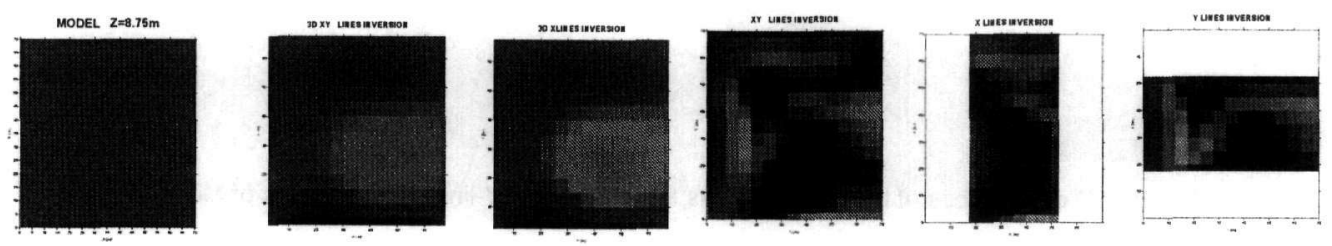

Figure 10: Depth slices of the 2D and 3D inversions for the $X$-lines, $Y$-lines and $X Y$-lines of Model 3 


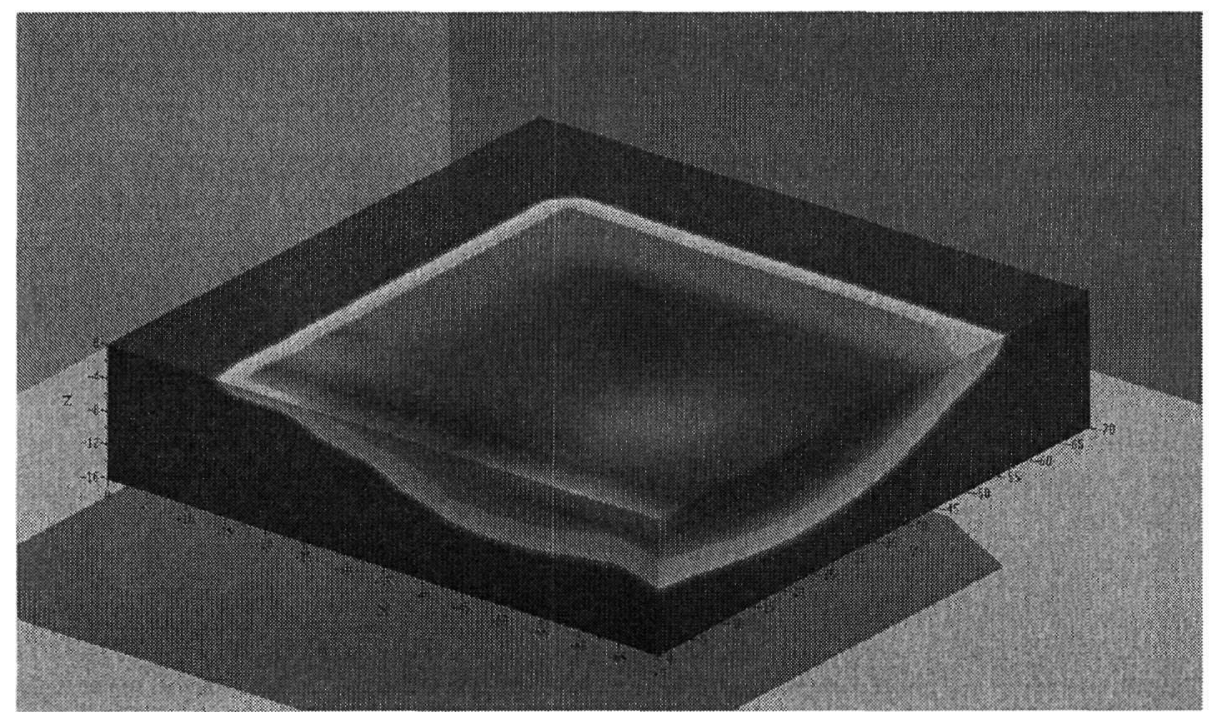

Figure 11: 3D view of the $X$-lines $3 \mathrm{D}$ inversion results for model 3

\section{REFERENCES}

Chambers J.E., 2001. The application of 3D electrical tomography to the investigations of brownfield sites. PhD Thesis, University of Sheffield.

Constable, S. Parker, R., \& Constable C., 1987. Occam's inversion: A practical algorithm for generating smooth models from electromagnetic sounding data. Geophysics, 52:289-300.

deGroot-Hedlin, C., and Constable, S., 1990.Occam's inversion to generate smooth, two-dimensional models from magnetotelluric data. Geophysics, 55:1613-1624.

Ellis, R., \& Oldenburg D.W., 1994. Applied geophysical inversion.Geophys.J.Int.,116, 5-11.

Loke M.H. and Barker R.D.,1996a. Rapid least-squares inversion of apparent resistivity pseudosections using a quasi-Newton method. Geophysical Prospecting, 44, 131-152.

Loke M.H. and Barker R.D.,1996b. Practical techniques for 3D resistivity surveys and data inversion. Geophysical Prospecting, 44, 499-523.

McGillivray, P., \& Oldenburg, D., 1990, Methods for calculating Frechet derivatives and sensitivities for the nonlinear inverse problem: A comparative study: Geophysical Prospecting, 38, 499-524.

Park, S.K. \& Van, G.P. 1991. Inversion of pole-pole data for 3-D resistivity structures beneath arrays of electrodes. Geophysics, 56, 951-960.

Sasaki, Y., 1989. 2-D joint inversion of magnetotelluric and dipole-dipole resistivity data. Geophysics, 54:254262.

Sasaki, Y., 1992. Resolution of resistivity tomography inferred from numerical simulation. Geophysical Prospecting, 40:453-464.

Tsourlos P., 1995. Modelling interpretation and inversion of multielectrode resistivity survey data. Ph.D. Thesis, University of York.

Tsourlos, P., Szymanski, J. \& Tsokas G., 1999, The effect of terrain topography on commonly used resistivity arrays: Geophysics, 64, 1357-1363.

Tsourlos P. \& Ogilvy R. 1999. An algorithm for the 3-D Inversion of Tomographic Resistivity and Induced Polarisation data: Preliminary Results. Journal of the Balkan Geophysical Society, 2, 2, 30-45.

Xu, B., 1993. Development of electrical resistivity imaging methods for geological and archaeological prospecting. Ph.D. Thesis, University of Durham 